Article

\title{
Entangled States in Quantum Cosmology and the Interpretation of $\Lambda$
}

\section{Salvatore Capozziello ${ }^{1, \star}$ and Orlando Luongo ${ }^{2,3}$}

${ }^{1}$ Dipartimento di Scienze Fisiche, Università di Napoli Federico II, Compl. Univ. di Monte S. Angelo, Edificio G, Via Cinthia, I-80126 Napoli, Italy

${ }^{2}$ Dipartimento di Fisica, Università di Roma La Sapienza, I-00185 Roma, Italy;

E-Mail: luongorl@roma1.infn.it

${ }^{3}$ ICRANet and ICRA (International Center of Relativistic Astrophysics Networks), Piazzale della Repubblica 10, I-65122 Pescara, Italy

* Author to whom correspondence should be addressed; E-Mail: capozziello@na.infn.it.

Received: 13 December 2010; in revised form: 2 February 2011 / Accepted: 16 February 2011 / Published: 17 February 2011

\begin{abstract}
The cosmological constant $\Lambda$ can be achieved as the result of entangled and statistically correlated minisuperspace cosmological states, built up by using a minimal choice of observable quantities, i.e., $\Omega_{m}$ and $\Omega_{k}$, which assign the cosmic dynamics. In particular, we consider a cosmological model where two regions, corresponding to two correlated eras, are involved; the present universe description would be, in this way, given by a density matrix $\hat{\rho}$, corresponding to an entangled final state. Starting from this assumption, it is possible to infer some considerations on the cosmic thermodynamics by evaluating the Von Neumann entropy. The correlation between different regions by the entanglement phenomenon results in the existence of $\Lambda$ (in particular $\Omega_{\Lambda}$ ) which could be interpreted in the framework of the recent astrophysical observations. As a byproduct, this approach could provide a natural way to solve the so called coincidence problem.
\end{abstract}

Keywords: quantum cosmology; entanglement; cosmological constant

PACS Codes: 98.80.-k, 98.80.Jk, 98.80.Cq, 98.80.Es 


\section{Introduction}

The quantization of gravity is still an open question which seems far to be achieved from several viewpoints [1]. For example, considering the canonical approach, the normalization and interpretation of the wave function, the meaning of time [2] are puzzles difficult to be framed in coherent and self-consistent schemes. These problems are directly due to the interpretation and the solution of the Wheeler-De Witt equation [3], and more generally, due to the way in which one quantizes space-time.

Some theoretical efforts have been proposed to solve these difficulties, see for example [4], but it seems that we are far from a definitive solution. Hence, a complete quantum description of General Relativity (GR) could be not possible.

On the other hand, several issues remain unsolved in modern cosmology, such as the problems of $d$ ark matter and dark energy [5]. Moreover, it seems that there are a lot of ways to interpret the cosmological constant $\Lambda$ which has several implications in the interpretation of observations [6].

Quantum cosmology, instead, has the final goal to describe the universe itself by methods and results of quantum theory. This implies that the universe as a whole should have a quantum description resulting in the "classical" observed cosmological parameters.

This fact can be viewed as a sort of entanglement with macroscopic degrees of freedom that leads to the classical appearance of macroscopic systems (in this case, the whole observed universe), a process known as de-coherence [7].

Hence a link between quantum information and quantum cosmology can be easily found considering the role of gravity. Indeed, quantum cosmology, being nothing else but an approximation, cannot be considered the full quantum gravity, but it can assume a key role to describe the observed state of the universe in the light of quantum information. This will be the main issue on which this paper is focused. In particular this point of view would allow us to explain, in the context of de-coherence and entanglement, some open questions of modern cosmology as the cosmological constant problem.

While quantum cosmology and quantum gravity have many interpretative problems, indeed, quantum information theory has recently increased its theoretical self-consistency producing several interesting results. The most important one has been the achievement that entanglement phenomena of quantum states have been framed in robust theoretical schemes and verified through several experimental tests (see for example [8-18]).

The idea of entanglement starts from the apparent conflict between the superposition principle and the non-separability of the related quantum states. It appears when a state of two or more subsystems of a compound quantum system cannot be factorized into pure local states of the subsystems too. This is equivalent to say that a state, which describes a particular physical system, could be thought as due to two or more than two sub-states, derived from subsystems, which are connected between them by a non-factorizing property [19]. To fix the ideas, if we take into account a state like

$$
|\Psi\rangle=\left|\Psi^{(1)}\right\rangle\left|\Psi^{(2)}\right\rangle
$$

this is well-factorized into two sub-states, i.e., $\left|\Psi^{(1)}\right\rangle$ and $\left|\Psi^{(2)}\right\rangle$; consequently, the state is not entangled, but a state like

$$
|\Psi\rangle=\sum_{n=0}^{M}\left|\Psi_{n}^{(1)}\right\rangle\left|\Psi_{n}^{(2)}\right\rangle
$$


is an entangled one. This suggests that a good way to study the complete state is a description by the density matrix $\hat{\rho}$ where one has to prepare the state $\left|\Psi_{1}\right\rangle$ with probability $p_{1}$, the state $\left|\Psi_{2}\right\rangle$ with probability $p_{2}$ and so on for all the sub-states of the whole system.

In order to understand the phenomenology of the entanglement, let us consider how it can be realized. When two physical systems come into interaction, some correlation of quantum nature is generated between them and persists also if any apparent interaction is not present and the two systems are spatially far [20]. Let us suppose that one has a bipartite (or multipartite) quantum state [21-23]: The non-separability or non-factorizability is necessary to take into account correlations [24-26] which can evolve with time.

Several different criteria are possible to characterize entanglement but all of them are essentially based on equivalent forms of non-locality in pure quantum states [27].

Hence, entanglement represents a fundamental physical resource [28] and it has become comparable with the ideas of energy or entropy in the information theory of systems.

Due to these motivations, the so-called entanglement-degree has become an important feature (see for example [21,29]) to quantify how much a quantum state is mixed or pure [22,30-32]. In this paper, we are going to show that the thermodynamics of the universe and then the emergence of the cosmological constant $\Lambda$ are both related to the entanglement-degree.

The paper is organized as follows. Section II is a brief summary of the entanglement theory. Section III is devoted to the definition of the pure and mixed cosmological states. We show that the evaluation of the Shannon-Von Neumann entropy $S$ [33] can be directly related to the emergence of a quantity which can be naturally considered the cosmological constant $\Lambda$. Conclusions are drawn in Section IV.

\section{Entanglement and Quantum Cosmology}

In order to develop considerations on the role of the entanglement in quantum cosmology, let us start with taking into account an $N$-dimensional Hilbert space where the probabilities are $p_{k}=\frac{1}{N}, \forall k$. The interpretation is that a given state is maximally mixed, or physically speaking, it means that our ignorance about the configuration is total.

The opposite situation, in other words the case in which only one of the $p_{k}$ is different from zero, happens when we have pure quantum states. The first step to quantify this situation is the introduction of a density matrix $\hat{\rho}$ associated to the quantum states. For a generic pure state $|\Psi\rangle$, the density matrix is the projector

$$
\hat{\rho}_{p}=|\Psi\rangle\langle\Psi|
$$

The main properties of $\hat{\rho}$ are easily given:

1. $\operatorname{Tr} \hat{\rho}=1$,

2. $\operatorname{Tr} \hat{\rho}^{2} \leq 1$,

3. $\langle\chi|\hat{\rho}| \chi\rangle \geq 0$.

It is worth noticing that $\operatorname{Tr} \rho^{2}=1$ holds only if the state is pure, vice versa $\operatorname{Tr} \rho^{2}<1$. For our aims, we will consider not pure states and then $\hat{\rho}$ becomes the sum on projectors, balanced by weights, which represents probabilities for mixed states [29]. 
As a consequence, we define the purity of a state the quantity

$$
\mu[\hat{\rho}]=\operatorname{Tr} \hat{\rho}^{2}
$$

and then the basic concept of linear entropy

$$
S_{L}=\frac{N}{N-1}(1-\mu[\hat{\rho}])
$$

which are two prototypes of entanglement measurements.

In particular, Equation (5) can be interpreted as a first-order approximation of the concept of entropy in the information theory, the so-called Von Neumann entropy, which usually describes how large is the lack of information of a quantum state [34]. We are interested to the thermal entanglement properties of the universe. In agreement with the statistical picture, we need a measure of the entropy of the system. The process of entropy measurement is related to the concept of the so called Shannon-Von Neumann entropy

$$
S=-\operatorname{Tr}(\hat{\rho} \ln \hat{\rho})=-\sum_{k} \lambda_{k} \ln \lambda_{k}
$$

which satisfies concavity, subadditivity and Araki-Lieb inequality (see for details [29]). The arbitrary choice of states on which the trace of the above equation can be evaluated, allows us to consider the eigenvalues $\lambda_{k}$ in Equation (6).

In this picture, the idea of entangled states in quantum cosmology assume an important role. We can take into account a cosmological model where all the eras are linked together by entanglement and then describe the thermodynamics of such entangled states.

This picture can be achieved starting from the consideration that the entropy $S$ of a system is a macroscopic quantity which can be determined without knowing in details the constituent microscopic states. In other words, a measure of entanglement is a "coarse grained" approach capable of contributing to the global description of the whole system.

The well-known problem of defining different vacuum states in quantum field theory could be overcome considering $\Lambda$ as the measure of the entanglement-degree related to the thermodynamical state of the universe. In other words, $\Omega_{\Lambda}$ at a given era can be considered as the result of the correlation between cosmological "quantum" states without knowing in details the pure states which contribute to the correlation itself.

\section{The Cosmological States and the Problem of $\Lambda$}

Let us take into account a set of observable quantities defining a cosmological state written as a vector

$$
\Upsilon=\left\{H(z), a(z), q(z), j(z), s(z), l(z), \Omega_{m}(z), \Omega_{k}(z), \Omega_{\Lambda}(z), T(z), \vec{o}(z)\right\}
$$

where $H(z)$ is the Hubble parameter [35], $q(z), j(z), s(z)$ are the cosmographic parameters, respectively the deceleration, the jerk and the snap [36-39], $\Omega_{m}$ is the matter density, $\Omega_{k}$ is spatial curvature density [40], $T$ is the temperature of CMB [33] and $\vec{o}$ is a subset of observable quantities which contribute to better determine the cosmological state. All the parameters are assumed as function of the redshift $z$ and univocally assign a cosmological state. We are simply assuming a minisuperspace model 
where cosmological principle holds and "evolution" means correlation among states. In some sense, we are defining cosmographies at given eras (the redshift $z$ ) and we are assuming that they are correlated without specifying a priori a field theory. It means that no theoretical model is involved when we, for example, measure $q(z)$; in fact, following this argument, we note that the experimental framework is the set of Supernovae data [41], or, again, $\Omega_{m}$ is measured by the abundance of matter in galaxies and so on for the other quantities in the $\Upsilon$ set [35]. In the vector $\Upsilon$, any parameters capable of describing the universe dynamics and thermodynamics can be considered. However the more the number of parameters increases, the more the cosmic state is better assigned but the more the phase-space increases in the number of dimensions. At this point the question is how to build up a description of universe, considering this set, in terms of quantum states. In other words, we are wondering if it is possible to achieve a quantum description of universe, starting from the quantum theory, without assigning a priori field theory like GR. In this way, we do not need any quantization of gravity [40,42-45], because the framework of GR is based on a quantum field theory; we require, so, no field quantization but only a quantum description of the universe, as a vector set, derived from (7).

It is only necessary to consider a set of astrophysical observables to infer the universe dynamics which could be achieved by entanglement measures. This minimal choice of observable quantities can be used to build up a complete phase-space evolution representing the cosmic dynamics. Moreover, the sense of "minimal choice" is that, in order to get a complete description of the state at a given redshift $z$, it is necessary to define quantities which are not linked, or parameterized among them. This allows, in principle, to achieve a description of the universe where variables are independent among them.

Because of $z$-dependence of all the parameters in the vector (7), z becomes not directly necessary in the set; moreover, assuming that any coarse-grained cosmological dynamics could be represented by a Friedmann-Roberson-Walker metric (FRW), the dimensionality of the vector can be reduced to two [46]. A minimal choice is then

$$
\left|\phi_{i}\right\rangle \equiv\left(\begin{array}{c}
\Omega_{m i} \\
\Omega_{k i}
\end{array}\right)
$$

where we do not consider yet the $z$ dependence but we labelled the quantities by $i=1,2$, which means that cosmological state is assigned by dynamical and thermodynamical parameters connecting two given regions (i.e., epochs). The way in which we link the two regions between them is the entanglement; in other words, we imagine that the epoches are correlated between them by entanglement. A mixing hypothesis appears now as another fundamental feature: All the regions are characterized by the fact that they are mixed with all the others; this will be clear as soon as we will take into account the density matrix description for universe states.

It is worth noticing that we deal only with two regions because we describe the simplest toy model able to show how entanglement, starting from very basic quantum assumptions could naturally account for macroscopic observable.

Let us consider now two regions and, hereafter, we will refer to the entangled states ansatz (ESA); this hypothesis enables us to build up a complete state of universe, defined first as a mixed one only and then, as an entangled one, from Equation (8). This means that a physical state of the universe may derive from an entanglement phenomenon between states at different eras. 
In order to construct a superposition of states, we must require that, for $i=1,2$, the "good" states at given eras are to be written in form of a basis in order to describe a complete entangled state. Following the Gram-Schmidt construction of orthogonal and normalized basis, we have

$$
\left|e_{1}\right\rangle=N_{1}\left(\begin{array}{c}
\Omega_{m 1} \\
\Omega_{k 1}
\end{array}\right)
$$

which is the first unitary vector; the other one is built up by considering the rule, (without normalization)

$$
\left|\tilde{e}_{2}\right\rangle=\left(\begin{array}{c}
\Omega_{m 2} \\
\Omega_{k 2}
\end{array}\right)-N_{1}^{2}\left(\begin{array}{ll}
\Omega_{m 1} & \Omega_{k 1}
\end{array}\right) \cdot\left(\begin{array}{c}
\Omega_{m 2} \\
\Omega_{k 2}
\end{array}\right)\left(\begin{array}{c}
\Omega_{m 1} \\
\Omega_{k 1}
\end{array}\right)
$$

or equivalently, here with normalization

$$
\left|e_{2}\right\rangle=N_{2}\left(\begin{array}{c}
\Omega_{m 2}-N_{1}^{2}\left(\Omega_{m 1}^{2} \Omega_{m 2}+\Omega_{m 1} \Omega_{k 1} \Omega_{k 2}\right) \\
\Omega_{k 2}-N_{1}^{2}\left(\Omega_{k 1}^{2} \Omega_{k 2}+\Omega_{m 1} \Omega_{k 1} \Omega_{m 2}\right)
\end{array}\right)
$$

where the normalization parameters $N_{1}$ and $N_{2}$ are given by

$$
\begin{aligned}
N_{1} & \equiv \frac{1}{\sqrt{\Omega_{m 1}^{2}+\Omega_{k 1}^{2}}} \\
N_{2} & \equiv \frac{1}{\sqrt{\left\{\Omega_{m 2}-N_{1}^{2}\left(\Omega_{m 1}^{2} \Omega_{m 2}+\Omega_{m 1} \Omega_{k 1} \Omega_{k 2}\right)\right\}^{2}+\left\{\Omega_{k 2}-N_{1}^{2}\left(\Omega_{k 1}^{2} \Omega_{k 2}+\Omega_{m 1} \Omega_{k 1} \Omega_{m 2}\right)\right\}^{2}}}
\end{aligned}
$$

Starting from this construction, one is able to have entanglement between the set of basis, postulating an entangled state, similar to the construction of a Bell state [16].

\subsection{The Entangled States Ansatz}

The ESA suggests that a possible state for describing the universe is

$$
\left|\Psi_{ \pm}\right\rangle=\alpha\left|e_{1} e_{1}\right\rangle \pm \beta\left|e_{2} e_{2}\right\rangle
$$

with the direct expression for $\left|e_{i} e_{i}\right\rangle$

$$
\left|e_{i} e_{i}\right\rangle=N_{i}^{2}\left(\begin{array}{c}
\Omega_{m i}^{2} \\
\Omega_{m i} \Omega_{k i} \\
\Omega_{m i} \Omega_{k i} \\
\Omega_{k i}^{2}
\end{array}\right)
$$

and the normalization given by

$$
|\alpha|^{2}+|\beta|^{2}=1
$$

in a particular Hilbert space, $\mathcal{H}$ with $\operatorname{dim} \mathcal{H}=4$ (such a number is not related to the number of physical dimensions but with the minimal parameters to "assign" a FRW universe in two eras. This is true, in terms of the Equation (14), if we define the two simplest positions:

$$
\Omega_{m 2}^{*}=\Omega_{m 2}-N_{1}^{2}\left(\Omega_{m 1}^{2} \Omega_{m 2}+\Omega_{m 1} \Omega_{k 1} \Omega_{k 2}\right) \rightarrow \Omega_{m 2}
$$


and

$$
\Omega_{k 2}^{*}=\Omega_{k 2}-N_{1}^{2}\left(\Omega_{k 1}^{2} \Omega_{k 2}+\Omega_{m 1} \Omega_{k 1} \Omega_{m 2}\right) \rightarrow \Omega_{k 2}
$$

Hereafter, in order to derive simple expressions for the next calculations, we use notation in (14); the state of that expression is coincident with the product of two states of the form of Equation (9) for $i=1$, but for $i=2$ it appears possible, only if the above simplest positions hold.

Note that $\Omega_{m 2}$ and $\Omega_{k 2}$ are functions of $\Omega_{m 1}, \Omega_{k 1}, \Omega_{m 2}$ and $\Omega_{k 2}$, differently from $\Omega_{m 1}, \Omega_{k 1}$, which are independent variables deduced, in principle, from observations at given eras.

A maximally entanglement phenomenon is present if $\alpha=\beta=\frac{1}{\sqrt{2}}$ up to a phase factor. Note that in our picture, it is not necessary to stress that $\left|e_{i} e_{i}\right\rangle=\left|e_{i A}\right\rangle\left|e_{i B}\right\rangle$, as mixed hypothesis suggests, because at the same era we may imagine that $\Omega_{\chi k A}=\Omega_{\chi k B}$, with $\chi=m, k$ and for $k=1,2$, to simplify the model.

Considering the density matrix $\hat{\rho}=\sum_{j= \pm} p_{j}\left|\phi_{j}\right\rangle\left\langle\phi_{j}\right|$, we have

$$
\begin{gathered}
\hat{\rho}=|\alpha|^{2}\left|e_{1} e_{1}\right\rangle\left\langle e_{1} e_{1}\left|\left(p_{+}+p_{-}\right)+2\left(\alpha \beta^{*}+\alpha^{*} \beta\right)\left(p_{+}-p_{-}\right)\right| e_{1} e_{1}\right\rangle\left\langle\left. e_{2} e_{2}|+| \beta\right|^{2} \mid e_{2} e_{2}\right\rangle\left\langle e_{2} e_{2}\right|\left(p_{+}+p_{-}\right) \\
=|\alpha|^{2}\left|e_{1} e_{1}\right\rangle\left\langle\left. e_{1} e_{1}|+| \beta\right|^{2} \mid e_{2} e_{2}\right\rangle\left\langle e_{2} e_{2}\left|+\left(\alpha \beta^{*}+\alpha^{*} \beta\right)\left(p_{+}-p_{-}\right)\right| e_{1} e_{1}\right\rangle\left\langle e_{2} e_{2}\right|
\end{gathered}
$$

Hence, after straightforward calculations, we get

$$
\begin{aligned}
& \hat{\rho} \equiv|\alpha|^{2} N_{1}^{2}\left(\begin{array}{cccc}
\Omega_{m 1}^{4} & \Omega_{m 1}^{3} \Omega_{k 1} & \Omega_{m 1}^{3} \Omega_{k 1} & \Omega_{m 1}^{2} \Omega_{k 1}^{2} \\
\Omega_{m 1}^{3} \Omega_{k 1} & \Omega_{m 1}^{2} \Omega_{k 1}^{2} & \Omega_{m 1}^{2} \Omega_{k 1}^{2} & \Omega_{m 1} \Omega_{k 1}^{3} \\
\Omega_{m 1}^{3} \Omega_{k 1} & \Omega_{m 1}^{2} \Omega_{k 1}^{2} & \Omega_{m 1}^{2} \Omega_{k 1}^{2} & \Omega_{m 1} \Omega_{k 1}^{3} \\
\Omega_{k 1}^{2} \Omega_{m 1}^{2} & \Omega_{k 1}^{3} \Omega_{m 1} & \Omega_{k 1}^{3} \Omega_{m 1} & \Omega_{k 1}^{4}
\end{array}\right)+ \\
& \times\left(\begin{array}{cccc}
\Omega_{m 1}^{2} \Omega_{m 2}^{2} & \Omega_{m 1}^{2} \Omega_{m 2} \Omega_{k 2} & \Omega_{m 1}^{2} \Omega_{m 2} \Omega_{k 2} & \Omega_{m 1}^{2} \Omega_{k 2}^{2} \\
\Omega_{m 1} \Omega_{k 1} \Omega_{m 2}^{2} & \Omega_{m 1} \Omega_{k 1} \Omega_{m 2} \Omega_{k 2} & \Omega_{m 1} \Omega_{k 1} \Omega_{m 2} \Omega_{k 2} & \Omega_{m 1} \Omega_{k 1} \Omega_{k 2}^{2} \\
\Omega_{m 1} \Omega_{k 1} \Omega_{m 2}^{2} & \Omega_{m 1} \Omega_{k 1} \Omega_{m 2} \Omega_{k 2} & \Omega_{m 1} \Omega_{k 1} \Omega_{m 2} \Omega_{k 2} & \Omega_{m 1} \Omega_{k 1} \Omega_{k 2}^{2} \\
\Omega_{k 1}^{2} \Omega_{m 2}^{2} & \Omega_{k 1}^{2} \Omega_{m 2} \Omega_{k 2} & \Omega_{k 1}^{2} \Omega_{m 2} \Omega_{k 2} & \Omega_{k 1}^{2} \Omega_{k 2}^{2}
\end{array}\right)+ \\
& +|\beta|^{2} N_{2}^{2}\left(\begin{array}{cccc}
\Omega_{m 2}^{4} & \Omega_{m 2}^{3} \Omega_{k 2} & \Omega_{m 2}^{3} \Omega_{k 2} & \Omega_{m 2}^{2} \Omega_{k 2}^{2} \\
\Omega_{m 2}^{3} \Omega_{k 2} & \Omega_{m 2}^{2} \Omega_{k 2}^{2} & \Omega_{m 2}^{2} \Omega_{k 2}^{2} & \Omega_{m 2} \Omega_{k 2}^{3} \\
\Omega_{m 2}^{3} \Omega_{k 2} & \Omega_{m 2}^{2} \Omega_{k 2}^{2} & \Omega_{m 2}^{2} \Omega_{k 2}^{2} & \Omega_{m 2} \Omega_{k 2}^{3} \\
\Omega_{k 2}^{2} \Omega_{m 2}^{2} & \Omega_{k 2}^{3} \Omega_{m 2} & \Omega_{k 2}^{3} \Omega_{m 2} & \Omega_{k 2}^{4}
\end{array}\right)
\end{aligned}
$$


where the normalized probability condition

$$
p_{+}+p_{-}=1
$$

has been used. From a physical point of view, it represents a constraint on the final state of the universe relating two eras. In fact, $p_{+}$is the probability that, the mixture representing the universe entangled state is in the configuration $\left|\Psi_{+}\right\rangle\left\langle\Psi_{+}\right|$and, on the other hand, $P_{-}$is the probability that, the universe mixture entangled state, is in the configuration $\left|\Psi_{-}\right\rangle\left\langle\Psi_{-}\right|$. This is compatible with probability normalization and with ESA. As reported in the Introduction, $\operatorname{Tr} \hat{\rho}=1$ is an invariant expression that can be used with other constraints to have a complete picture of the quantum nature of universe. For maximally entangled states and real $\alpha, \beta$

$$
\frac{t_{1}\left(\Omega_{m 2}^{2} \Omega_{m 1}^{2}+\Omega_{k 1}^{2} \Omega_{k 2}^{2}\right)+t_{2} \Omega_{m 1} \Omega_{m 2} \Omega_{k 1} \Omega_{k 2}+\Omega_{k 2}^{2}\left(\Omega_{m 1}^{2}+\Omega_{k 1}^{2}\right)}{2\left(\Omega_{m 1}^{2}+\Omega_{k 1}^{2}\right)\left(\Omega_{m 2}^{2}+\Omega_{k 2}^{2}\right)}=1
$$

where $t_{1}=4 \alpha \beta-8 \alpha \beta p_{-}+1$ and $t_{2}=-8 \alpha \beta\left(1-2 p_{-}\right)$with the conditions that $\alpha$ and $\beta$ are real and the states are maximally entangled.

In addition, it is important to note that, because of $\Omega_{m h}+\Omega_{k h}<1$ coming from the astrophysical observations at all the epoches (i.e., $\forall h=1,2$ ), we can reduce to a constraint such inequality on $\Omega_{m i, k i}$, that is

$$
\left\{\begin{array}{l}
\Omega_{m 1}+\Omega_{k 1}+\sum_{i} \Omega_{X 1 i}=1 \\
\Omega_{m 2}+\Omega_{k 2}+\sum_{j} \Omega_{X 2 j}=1
\end{array}\right.
$$

Immediately we can imagine a straightforward interpretation of it. Because of $\Omega_{m i}<1, \Omega_{k i}<1$, some unknown quantities are involved into calculations. All these parameters, in particular $\Omega_{X h k}(z)$, are functions of the redshift $z$ being functions of the scale factor $a(t)$. Equations (19) are a natural constrain since they are nothing else but the Friedmann equation at different $z$. To be more precise, if we require that the constraint is based on observable quantities, i.e., $\Omega_{m}$ and $\Omega_{k}$, we have no reason to think that the $\Omega_{X h k}(z)$ have to be different between them. This suggests $\Omega_{X h k}(z)=\Omega_{X k}(z)$. The nature of it is, at the same time, linked to the previous two densities; in this sense it is useful to note that no dependence from $z$ is necessary, since if $\Omega_{m}$ and $\Omega_{k}$ vary with $z$, by conservation relations, it is not the simplest way to think that the sum of them loses a part of mass or curvature in time, in some unknown variables as $\Omega_{X k}(z)$. It may be a nonsense. Hence, from this, it is compatible with the choice of $\Omega_{X k}(z)=\Omega_{X k}$ and $\Omega_{X k} \in[0,0.3]$. By considering Equation (19), it is natural to imagine that we are assuming a given cosmological model able to constrain to one the above sums. This is what happens if 0,0-Einstein-Friedmann equation is taken into account in cosmological standard model. However, because of the definitions of entanglement, entropy and related probabilities, all quantities are strongly normalized to one. This statement is equivalent to claim that the sums of observable quantities must be normalized and the loss of information due to the entanglement process, which is at the basis of ESA, must be added in the term of $\Omega_{\Lambda}$. In other words, ESA is consistent with cosmological Einstein equations if cosmological term is present into dynamics.

If we recall $\sum_{k} \Omega_{X k}$ with the compact name of $\Omega_{\Lambda}$, we conclude that: We consider a constraint which is the fixed normalization of observable quantities energy-matter and curvature density, given in principle, by a sum of unknown quantities. If these quantities are summing up and defined as one entity, 
it has to be considered as independent of redshift $z$ and with no different behavior in the different eras of universe evolution (that is, at the same time, compatible with the universe dynamics).

Because of the given meaning of $\Omega_{\Lambda}$, it is possible to connect its existence with the lack of information of an entangled state, described by the Von Neumann entropy, as discussed. By construction of it, in fact, the role of eigenvalues and of entropy is the key to understand the existence of $\Omega_{\Lambda}$ density. Note that, up to now, no cosmological constant model is proposed, in terms of an added cosmological constant into account of GR, because GR has not entered the ESA. This is important because tries to understand the specific role of $\Omega_{\Lambda}$ density, without passing through a (classical or quantum) field theory.

Each epoch, in particular, has got its own reduced density matrix, so its own quantum description, as part of $\hat{\rho}$, describing also its own dynamics. A similar matrix is defined as

$$
\hat{\rho}^{A}=\operatorname{Tr}_{B} \hat{\rho}
$$

in fact $\hat{\rho}=\hat{\rho}^{A B}$, because is defined, in principle, on two different spaces, $A$ and $B$, which correspond to parts of the two eras, characterized by different $z$ evolutions. The expression of reduced density reads [47] $\hat{\rho}^{A}=\hat{\rho}^{B}=|\alpha|^{2}\left|e_{1}\right\rangle\left\langle\left. e_{1}|+| \beta\right|^{2} \mid e_{2}\right\rangle\left\langle e_{2}\right|$.

The eigenvalues [48] calculation of density matrix $\hat{\rho}$ allows us to write down entropy as follows

$$
S=-\left(\lambda_{3} \ln \lambda_{3}+\lambda_{4} \ln \lambda_{4}\right)
$$

which is a direct consequence of the arbitrary choice of the state in evaluating the trace. The complete forms of eigenvalues are very difficult to write down; to fix the ideas, in the case of $\Omega_{k 1} \approx 0$, for $p_{+}=p_{-}[49]$, they read

$$
\begin{gathered}
\lambda_{1}=0 \\
\lambda_{2}=0 \\
\lambda_{3}=\frac{1}{2}\left(1+\frac{\sqrt{2 \Omega_{m 2}^{2} \Omega_{k 2}^{2}\left(\alpha^{2}-\beta^{2}\right)^{2}+\Omega_{m 2}^{4}+\Omega_{k 2}^{4}\left(\alpha^{2}-\beta^{2}\right)^{2}}}{\Omega_{m 2}^{2}+\Omega_{k 2}^{2}}\right) \\
\lambda_{4}=-\frac{1}{2}\left(1-\frac{\sqrt{2 \Omega_{m 2}^{2} \Omega_{k 2}^{2}\left(\alpha^{2}-\beta^{2}\right)^{2}+\Omega_{m 2}^{4}+\Omega_{k 2}^{4}\left(\alpha^{2}-\beta^{2}\right)^{2}}}{\Omega_{m 2}^{2}+\Omega_{k 2}^{2}}\right)
\end{gathered}
$$

For maximally entangled states, we infer the expression for $\Omega_{\Lambda}$, in the case of $\Omega_{k 1} \approx 0$ [50]

$$
\Omega_{\Lambda}=-\frac{\omega_{a} \Omega_{m 1}^{5}-\omega_{b} \Omega_{m 1}^{4}-2 \Omega_{m 1}^{3} \Omega_{m 2}^{2}+2 p_{+} \Omega_{m 1}^{2} \Omega_{m 2}^{2}+\Omega_{m 1} \Omega_{m 2}^{2}+2 p_{-} \Omega_{m 2}^{2}-\Omega_{m 2}^{2}}{\left(\Omega_{m 1}^{2}+\Omega_{k 1}^{2}\right) \Omega_{m 1}^{4}-\Omega_{m 2}^{2}\left(2 \Omega_{m 1}^{2}-1\right)}
$$

where $\omega_{a} \equiv \Omega_{m 2}^{2}+\Omega_{k 2}^{2}, \omega_{b} \equiv p_{-} \Omega_{m 2}^{2}-\Omega_{m 2}^{2}-\Omega_{k 2}^{2} / 2$. If the behavior of states is not maximally entangled and no significative approximations are done, then the most general expressions of $\Omega_{\Lambda}$, with minimal positions again, for brevity, reads 


$$
\Omega_{\Lambda}=\frac{-f_{1}\left(\Omega_{m}\right) \Omega_{m 1}^{3}-f_{2}\left(\Omega_{m}\right) \Omega_{m 1}^{2}+f_{3}\left(\Omega_{m}\right) \Omega_{m 1}-f_{1}\left(\Omega_{m}\right) \Omega_{k 1}^{3}+f_{4}\left(\Omega_{m}\right) \Omega_{k 1}^{2}}{\left(\Omega_{m 1}^{2}+\Omega_{k 1}^{2}\right)\left(\Omega_{m 2}^{2}+\Omega_{k 2}^{2}\right)}
$$

where

$$
\begin{aligned}
& f_{1}\left(\Omega_{m}\right):=\Omega_{k 2}^{2}+\Omega_{m 2}^{2}, \\
& f_{2}\left(\Omega_{m}\right):=2 \Omega_{m 2}^{2} \alpha \beta p_{-}+\Omega_{k 1} \Omega_{m 2}^{2}-\alpha^{2} \Omega_{k 2}^{2}-2 \Omega^{2} \alpha \beta p_{-}-\Omega_{m 2}^{2} \alpha^{2}-\beta^{2} \Omega_{k 2}^{2}+\Omega_{k 1} \Omega_{k 2}^{2}-\Omega_{m 2}^{2} \beta^{2}, \\
& f_{3}\left(\Omega_{m}\right):=-\Omega_{k 1}^{2} \Omega_{k 2}^{2}-\Omega_{k 1}^{2} \Omega_{k 2}^{2}-4 \Omega_{k 2} \alpha \beta \Omega_{k 2} \Omega_{k 1} p_{-}+4 \Omega_{m 2} \alpha \beta \Omega_{k 2} \Omega_{k 1} p_{+}, \\
& f_{4}\left(\Omega_{m}\right):=\beta^{2} \Omega_{k 2}^{2}+\Omega_{m 2}^{2} \beta^{2}+\alpha^{2} \Omega_{k 2}^{2}+2 \alpha \beta p_{+} \Omega_{k 2}^{2}+\Omega_{m 2}^{2} \alpha^{2}-2 \alpha \beta p_{-} \Omega_{k 2}^{2} .
\end{aligned}
$$

The entanglement measurements are various and different as mentioned in the introduction. Allowing a variation of entropy, in terms of $\Omega_{m 2}$ and $\Omega_{k 2}$, we infer the grade of entanglement, having an idea of its variation with parameters of the second era.

\subsection{The Coincidence Problem and the Interpretation of $\Lambda$}

The coincidence problem is a puzzling situation which appears in today cosmology. It is essentially due to the resulting same order of magnitude for $\Omega_{m}$ and $\Omega_{\Lambda}$. From the previous discussion, it could be addressed by means of Equation (24) for $\Lambda$. It is evident, in fact, that the condition

$$
0<\Omega_{\Lambda}<1
$$

can be easily achieved and so, according to the observations, this could be seen as an explanation of the coincidence problem. To clarify this statement, let us imagine to perform an analysis in which the matter density of the first era is approximatively of the same order of the second one, in a quasi-flat Universe, i.e., $\Omega_{m i} \gg \Omega_{k i}, \forall i$; the numerator in Equation (24) is proportional to $\Omega_{m}^{5}$, while denominator to $\Omega_{m}^{4}$, then $\Omega_{\Lambda} \propto \Omega_{m}$. This result naturally provides the cosmological coincidence.

Indeed, because of the meaning of $\Omega_{\Lambda}$, induced by the entanglement, it is evident, for construction, that its order of magnitude is the same of $\Omega_{m}$ and $\Omega_{k}$. The interpretation of $\Lambda$ is the following:

The observed value of cosmological constant $\Lambda$ derives from entanglement phenomena among cosmological eras. It is the result of an average statistical process generated by the superposition of several cosmic quantum states. Astrophysical observations are measuring the result of such a superposition.

\section{Conclusions and Perspectives}

We have studied thermal properties of universe and interpreted the cosmological constant problem in view of the so-called entangled states ansatz (ESA). Hence the idea of two discrete states, corresponding to the measurements of mass and curvature density at a given era is presented; such states can result entangled giving rise to the cosmological constant. This view is alternative to the standard GR approach where $\Lambda$ is imposed by hand in the cosmic evolution. In particular, the proposed toy model would give a straightforward approach to connect the today observed (classical) universe to quantum cosmology. In a sense, also the today observed universe is a quantum state resulting from various entanglement processes. The role of Friedmann cosmology in this approach is to link the emerging value of $\Omega_{\Lambda}$ to the various 
epochs since cosmological parameters depend on the redshift $z$. In this picture, the ESA hypothesis suggests a precise role of a cosmological states, instead of standard techniques, where geometric and matter degrees of freedom have to be decomposed to achieve quantization in generic curved space-times [42-44,51-62].

By considering two cosmic eras and imposing that they are correlated by a minimal choice of observable quantities, it is possible to use the Von Neumann entropy $S$ as an information tool to evaluate such a correlation. Thermodynamics is recovered by assuming $S \rightarrow k_{B} S$ which means that the entropy of the universe is derived by quantum entangled states. The phenomenon of entanglement enters the model, in terms of statistical considerations [37]. The result is that the standard cosmological constant $\Lambda$ emerges from an average process.

Also coincidence problem is solved in this context: the present values of $\Omega_{m}$ and $\Omega_{\Lambda}$ result of the comparable order of magnitude assuming $\Lambda$ as emerging from an entanglement process. The next step in this program is to achieve all the observed cosmographic parameters as the result of entanglement. This could be a confirmation for the present approach since cosmography is, in general, a robust test bed for cosmological models $[63,64]$. The present paper, in this sense, is the starting point of this program.

Moreover, it seems possible to connect the statistical interpretation of $\Lambda$ with a genuine quantum field theory interpretation, since $\Lambda$ can be the eigenvalue of general relativistic Hamiltonians [45]. In this way, the ESA hypothesis could be fully framed in a canonical quantization procedure.

\section{References and Notes}

1. Hartle, J.B. The Quantum Structure of Space and Time. In Proceedings of the 23rd Solvay Conference on Physics, Brussels, Belgium, 1-3 December 2005.

2. Shestakova, T.P.; Simeone, C. The problem of time and gauge invariance in the quantization of cosmological models. Grav. Cosmol. 2004, 10, 257-268.

3. Hartle, J.B. Prediction In Quantum Cosmology. In Gravitation in Astrophysics: Cargese 1986; Springer: New York, NY, USA, 1986.

4. Christensen, S.M. The Quantum Structure of Space and Time; Duff, M.J., Isham, C.J., Eds.; Cambridge University Press: Cambridge, UK, 1982.

5. Copeland, E.J.; Sami, M.; Tsujikawa, S. Dynamics of dark energy. Int. J. Mod. Phys. D 2006, 15, 1753-1936.

6. Andrianov, A.A.; Cannata, F.; Giacconi, P.; Kamenshchik, A.Y.; Soldati, R. Symmetries and the cosmological constant puzzle. Phys. Lett. B 2007, 651, 306-312.

7. Joos, E.; Zeh, H.D.; Kiefer, C.; Giulini, D.J.W.; Kupsch, J.; Stamatescu, I.-O. Decoherence and the Appearance of a Classical World in Quantum Theory; Springer: Berlin, Germany, 2003.

8. Gühne, O.; Toth, G. Entanglement detection. Phys. Rep. 2009, 474, 1.

9. Van Enk, S.J.; Lutkenhaus, N.; Kimble, H.J. On experimental procedures for entanglement verification. Phys. Rev. A 2007, 75, 052318.

10. Carteret, H. Exact interferometers for the concurrence and residual 3-tangle. Quant. Phys. 2006, arXiv:quant-ph/0309212v6.

11. Horodecki, P. Measuring Quantum Entanglement without Prior State Reconstruction. Phys. Rev. Lett. 2003, 90, 167901. 
12. Horodecki, P.; Ekert, A. Direct detection of quantum entanglement Phys. Rev. Lett. 2002, 89, 127902.

13. Walborn, S.P.; Souto Ribeiro, P.H.; Davidovich, L.; Mintert, F.; Buchleitner, A. Experimental determination of entanglement with a single measurement. Nature 2006, 440, 1022-1024.

14. Schmid, C.; Kiesel, N.; Laskowski, W.; Wieczorek, W.; Zukowski, M.; Weinfurter, H. Discriminating multi-partite entangled states. Phys. Rev. lett. 2008, 100, 200407.

15. Vidal, G. Entanglement monotones. J. Mod. Opt. 2000, 47, 355.

16. Bell, J.S. Speakable and Unspeakable in Quantum Mechanics; Cambridge University Press: Cambridge, UK, 1987.

17. Clauser, J.; Horne, M.; Shimony, A.; Holt, R. Proposed Experiment to Test Local Hidden-Variable Theories. Phys. Rev. Lett. 1969, 23, 880-884.

18. Clauser, J.F.; Horne, M. Experimental consequences of objective local theories. Phys. Rev. D 1974, 10, 526-535.

19. Shannon, C.E. Entropy. Bell Syst. Tech. J. 1948, 27, 379-423, 623-656.

20. Einstein, A.; Podolsky, B.; Rosen, N. Can Quantum-Mechanical Description of Physical Reality Be Considered Complete? Phys. Rev. 1935, 47, 777-780.

21. Heiss, D. Fundamentals of Quantum Information; Heiss, D., Ed.; Springer-Verlag: Berlin, Germany, 2002.

22. Audenaert, K.M.R.; Plenio, M.B. When are correlations quantum? Verification and quantification of entanglement by simple measurements. New J. Phys. 2006, 8, 266.

23. Plenio, M.B.; Virmani, S. An introduction to entanglement measures. Quant. Inf. Comp. 2007, 7, $1-51$.

24. Bell, J.S. On the Problem of Hidden Variables in Quantum Mechanics Rev. Mod. Phys. 1966, 38, 447-452.

25. Freedman, S.J.; Clauser, J.F. Experimental Test of Local Hidden-Variable Theories. Phys. Rev. Lett. 1972, 28, 938-941.

26. Gisin, N. Bell's inequality holds for all non-product states. Phys. Lett. A 1991, 154, 201-202.

27. These equivalences fade when we deal with mixed states.

28. Facchi, P.; Florio, G.; Parisi, G.; Pascazio, S. Maximally multipartite entangled states. Phys. Rev. A 2008, 77, 060304.

29. Nielsen, M.A.; Chuang, I.L. Quantum Computation and Quantum Information; Cambridge University Press: Cambridge, UK, 2002.

30. Henderson, L.; Vedral, V.J. Classical, quantum and total correlations. J. Phys. A 2001, 34, 6899-6905.

31. Groisman, B.; Popescu, S.; Winter, A. On the quantum, classical and total amount of correlations in a quantum state. Phys. Rev. A 2005, 72, 032317.

32. Peres, A. Quantum Theory: Concepts and Methods; Kluwer, Achademic Publishers: Dordrecht, The Netherlands, 1993.

33. Padmanabhan, T. Theoretical Astrophysics; Cambridge University Press: Cambdridge, UK, 2002.

34. Van Enk, S.J. The joys of permutation symmetry: Direct measurements of entanglement. Phys. Rev. Lett. 2009, 102, 190503. 
35. Peebles, P.J.E. Principles of Physical Cosmology; Princeton University Press: Princeton, NJ, USA, 1993.

36. Hawking, S.W.; Penrose, R. The singularities of gravitational collapse and cosmology. Proc. $R$. Soc. Lond. A 1970, 314, 529-548.

37. Luongo, O.; Iannone, I.; Autieri, C. Scale-Free model for governing Universe dynamics. EPL 2010, 90, 39001.

38. Sahni, V.; Saini, T.D.; Starobinsky, A.A. Statefinder: A new geometrical diagnostic of dark energy. JETP Lett. 2003, 77, 201-206.

39. Alam, U.; Sahni, V.; Saini, T.D.; Starobinsky, A.A. Exploring the Expanding Universe and Dark Energy using the Statefinder Diagnostic. Mon. Not. Roy. Astron. Soc. 2003, 344, 1057-1074.

40. Misner, C.W.; Thorne, K.S.; Wheeler, J.A. Gravitation; Freeman and Company: New York, NY, USA, 1973.

41. Visser, M. Jerk, snap, and the cosmological equation of state. Class. Quant. Grav., 2004, 21, 2603-2616.

42. Arnowitt, R.; Deser, S.; Misner, C.S. Dynamical structure and definition of energy in General Relativity. Phys Rev. 1959, 116, 1322-1330.

43. Arnowitt, R.; Deser, S. Canonical variables for General Relativity. Phys. Rev. 1960, 117, 1595-1602.

44. Arnowitt, R.; Deser, S. The Dynamics of General Relativity; John Wiley \& Sons: Oxford, UK, 1962.

45. Capozziello, S.; Garattini, R. The cosmological constant as an eigenvalue of $\mathrm{f}(\mathrm{R})$-gravity Hamiltonian constraint. Class. Quant. Grav. 2007, 24, 1627-1646.

46. It is easy to understand, considering the homogeneity and isotropy of the observed universe, that to describe cosmic dynamics, only two quantities are needed, for example $H$ and $\Omega_{m}$; the others, as for example the remaining cosmographic parameters, can be expressed in terms of them.

47. With equivalence between $A$ and $B$.

48. Note that $\lambda_{1} \ln \lambda_{1}=\lambda_{2} \ln \lambda_{2}=0$ because $\lambda_{1,2}=0$ and entropy in this case is defined $0 \ln 0=0$.

49. We do not expect that this condition is crucial and physically important; a mixture of external products among states, with the same weight, allows us only to simplify the $\hat{\rho}$ expression.

50. For the sake of simplicity, this result is written in the easy case of $p_{-}=\frac{1}{2}$ and, here, restoring, also, the definitions of $\Omega_{m 2}$ and $\Omega_{k 2}$, without the simplest positions.

51. Birrel, N.D.; Davies, P.C.W. Quantum Fields in Curved Space; Cambridge University Press: Cambridge, UK, 1982.

52. Wipf, A. Hamilton Formalism for Systems with Constraints. In Proceedsing of the The Canonical Formalism in Classical and Quantum General Relativity Seminar, Bad Honnef, Germany, 13-17 September 1993.

53. Govaerts, J.; Rashid, M.S. The Hamiltonian formulation of higher orders dynamical systems. High Energ. Phys. Theor. 1994, arXiv:hep-th/9403009v2.

54. Pons, J.M. Ostrogradsky theorem for higher order singular Lagrangians. Lett. Math. Phys. 1989, $17,181$.

55. Held, A. General Relativity and Gravitation; Plenum Press: New York, NY, USA, 1980. 
56. Madsen, M.S.; Barrow, J.D. De Sitter ground states and boundary terms in generalised gravity. Nucl. Phys. B 1989, 323, 242-252.

57. Hartle, J.B. Quantum Cosmology, Problems for the 21st Century. Gener. Relat. Quant. Cosmol. 1997, arXiv:gr-qc/9701022v2.

58. Hartle, J.B.; Hawking, S.W. Wave Function of the Universe. Phys. Rev. D 1983, 28, 2960-2975.

59. Halliwell, J.J.; Hartle, J.B. Integration contours for the no-boundary wave function of the universe. Phys. Rev. D 1990, 41, 1815-1834.

60. Hawking, S.W.; Hunter, C.J. The gravitational Hamiltonian, action, entropy and surface terms. Class. Quant. Grav. 1996, 13, 2735-2752.

61. Govaerts, J. Hamiltonian Quantisation and Constrained Dynamics (Leuven Notes in Mathematics and Theoretical Physics); Leuven University Press: Leuven, Belgium, 1991; Volume 4.

62. Ehlers, J.; Friedrich, H. Canonical Gravity: From Classical to Quantum (Lectures Notes on Physics); In Proceedings of the 117th WE Heraeus Seminar, Bad Honnef, Germany, 13-17 September 1993; Springer-Verlag: Berlin, Germany, 1994.

63. Capozziello, S.; Cardone, V.F.; Salzano, V. Cosmography of $\mathrm{f}(\mathrm{R})$ gravity. Phys. Rev. D 2008, 78, 063504.

64. Bouhmadi-Lopez, M.; Capozziello, S.; Cardone, V.F. Cosmography of $\mathrm{f}(\mathrm{R})$-Brane cosmology. Phys. Rev. D 2010, 82, 103526.

(c) 2011 by the authors; licensee MDPI, Basel, Switzerland. This article is an open access article distributed under the terms and conditions of the Creative Commons Attribution license (http://creativecommons.org/licenses/by/3.0/.) 